\title{
Du breton armoricain aux « celticismes »
}

From Armorican Breton to 'Celticisms'

\section{Francis Favereau}

\section{OpenEdition}

\section{Journals}

Édition électronique

URL : http://journals.openedition.org//bl/364

DOl : $10.4000 / / b l .364$

ISSN : 2727-9383

\section{Éditeur}

Université de Bretagne Occidentale - UBO

\section{Édition imprimée}

Date de publication : 1 octobre 2018

Pagination : 195-204

ISBN : 979-10-92331-40-0

ISSN : 1270-2412

\section{Référence électronique}

Francis Favereau, «Du breton armoricain aux « celticismes » », La Bretagne Linguistique [En ligne], 22 | 2018, mis en ligne le 01 mai 2020, consulté le 01 octobre 2020. URL : http://journals.openedition.org/ lbl/364; DOI : https://doi.org/10.4000/lbl.364

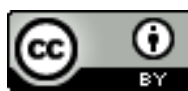

La Bretagne Linguistique est mise à disposition selon les termes de la Licence Creative Commons Attribution 4.0 International. 
Actualités

de la recherche 



\title{
Du breton armoricain aux « celticismes »
}

\author{
Francis FAVEREAU*
}

Pour répondre à l'invitation de Nelly Blanchard et de la revue $L a$ Bretagne linguistique à venir parler de mon récent ouvrage Celticismes - les Gaulois et nous ${ }^{1}$, je vais tenter de répondre aux deux questions qu'elle m'a posées - le pourquoi et le comment - sans autres prétentions, qu'elles soient méthodologiques ou théoriques, car même si c'est un brin paradoxal, je ne me considère pas comme un vrai spécialiste de la question, n'étant tout bonnement qu'un bretonnant de bonne volonté face à la question de l'origine de nos langues (celtiques et autres), celles qui nous entourent d'abord, même si je suis quand même, je l'avoue, un peu lexicographe du breton contemporain et si je me suis intéressé depuis un certain temps à l'étymologie, à ses tenants et aboutissants, comme d'autres se passionnent souvent pour leur généalogie, etc.

Il s'agit donc d'un travail de vulgarisation, partant de la re-

* Professeur émérite de langue et littérature bretonnes, CRBC Rennes 2. francis.favereau@wanadoo.fr

1. Francis FAVEREAU, Celticismes - les Gaulois et nous, Morlaix, Skol Vreizh, 2017. cherche appliquée, plutôt que de recherche "fondamentale", sur lesdites racines celtiques, présentes en nombre finalement dans les langues de notre environnement immédiat, sans oublier bien entendu l'aspect patrimonial qui concerne spécifiquement notre breton armoricain (dixit feu Hélias), témoin privilégié au titre du néo-celtique, car présent sur le continent depuis des millénaires, donc logiquement parmi les langues celtiques le mieux à même de témoigner d'une certaine continuité linguistique continentale sur deux millénaires et plus entre le celtique antique, puis ancien, et le breton armoricain dans ses diverses phases.

Paradoxalement, je ne me présente donc pas comme un philologue érudit spécialiste de la question comme nos illustres prédécesseurs, mais plutôt comme un lexicographe du breton actuel abordant l'aspect étymologique d'une façon pragmatique, sans a priori et sans non plus partir d'un présupposé théorique comme cela est souvent le cas, ni même d'un choix méthodologique défi- 
ni à l'avance. C'est plutôt en travaillant sur la durée - plusieurs années, voire quelques décennies depuis ma thèse (1984) - à l'étymologie du volumineux Dictionnaire du breton contemporain que j'ai récemment mis en ligne ${ }^{2}$, qu'il m'est apparu utile, partant d'un effet de puzzle quasi mécanique, d'aborder ces questions touchant à la linguistique de contact comme à la diachronie des langues celtiques.

Parallèlement il m'a paru évident, comme à d'autres, que le discours sur les origines de ces langues, comme des Celtes en général, ne correspondait absolument plus aux nombreuses avancées du dernier quart du $\mathrm{XX}^{\mathrm{e}}$ siècle, puis du XXI ${ }^{\mathrm{e}}$, comme le soulignent d'éminents spécialistes, tels Barry Cunliffe ou John Koch, etc. C'est pourquoi j'ai tenté également de faire un point sommaire sur le renouvellement de ces théories, comme celle de la continuité ou continuità (de l'Italien Alieni), abordée ici même à Brest il y a quelques années à propos du breton, d'autant que le renouvellement des recherches préhistoriques a débouché sur divers ouvrages de vulgarisation chez mon éditeur, sans que rien de parallèle n'y a été publié au plan linguistique. Comment s'étonner dès lors que le celtique soit totalement exclu des usuels, littéralement zappé

2. Voir http://GeriadurBrasFavereau. monsite-orange.fr (consulté le 18 juin 2018) par divers historiens spécialistes de la préhistoire de l'Armorique et que le vocable même de Celtes devienne suspect aux yeux de ces spécialistes ou soit, à tout le moins pour beaucoup, une construction d'aimables néo-celtomanes?

Par-delà les nombreux exemples issus de travaux antérieurs, depuis Loth ou Dottin, voire plus avant, en passant par Falc'hun et Fleuriot, jusqu'aux récentes publications de Lambert et Delamarre, abondamment reprises désormais sur Internet, souvent commune par commune dans toute la France et au-delà, je me suis parfois enhardi à suivre mon intuition ou plutôt ce que me suggérait tel ou tel rapprochement, avec la prudence extrême, presque de Sioux si l'on veut, qui s'impose en la matière.

Cette approche, qui s'apparente parfois davantage à l'archéologie qu'à la généalogie linguistique, n'est pas faite bien sûr que de certitudes et d'évidences. C'est pourquoi il faut toujours rester des plus humbles devant la contradiction, me semble-t-il, pour se montrer prêt à accepter la critique. Voilà autant de limites à une approche personnelle qui est ici davantage celle de l'essai - au sens premier - que du manuel, même si l'ouvrage relève pour l'essentiel d'un lexique thématique classique illustré de quelques centaines de termes restés dans les langues néo-celtiques, parfois dans des recoins ou replis divers, mais par- 
fois aussi passés largement dans les langues avoisinantes, petites et grandes.

C'est pour cela que je terminerai mon exposé en évoquant les limites de la démarche entreprise.

Ainsi ai-je osé relier, sans filet de sécurité à tort ou à raison, le terme « camion» (d'origine inconnue, selon divers dictionnaires) au second élément nominal d'un composé assez bien connu en gaulois et abondamment commenté, notamment par PierreYves Lambert, dont on connaît le brillant ouvrage sur la langue gauloise $^{3}:$ "(regu-)cambion" (au sens d'os courbes ou recourbés ici à redresser ou rebouter dans une formule de magie connue depuis longtemps).

S'appuyant sur le pluriel attesté de Cam (patronyme et forme galloise conservant le pluriel de l'adjectif -ion /-ien en breton actuel comme celui du patronyme courant (Le) Cam : Kammien), on peut le relier au composé bien connu en breton maritime koad-kamm, au sens des bordés en bois d'embarcation traditionnelle (ainsi à Douarnenez, relevé dans sa thèse par Per Denez), bois courbes, selon une technique d'ailleurs connue des anciens Celtes, notamment gaulois, relayé dans divers musées (comme celui de Saint-Germainen-Laye). Ces morceaux recourbés (koad kamm / kammion - ancien-

3. Pierre-Yves Lambert, La Langue gauloise, Paris, Errance 1994. nement camion en vieux-breton, toujours en gallois) auraient pu former les brancards d'une sorte d'éfourceau, ancêtre probable du fourgon tracté et bâché avant l'actuel " camion " industriel (terme par ailleurs présent en espagnol, notamment au Mexique où je l'ai entendu pour une sorte de bus, alors que le celtique carro s'y emploie à la place de l'espagnol coche, double illustration improbable mais parlante de cette odyssée des mots dans l'espace comme dans le temps).

Autre exemple incongru: le celtique ancien " argios ", assez bien attesté au sens de (blanc) brillant, nous a donné le terme breton erc' $^{\prime} h$ (neige), tout comme son équivalent gallois de même sens eira. Ce terme apparaît d'ailleurs ainsi, avec ces références, dans les éditions du Dictionnaire de la langue gauloise $^{4}$ de Xavier Delamarre. Si l'on fait abstraction du cas nominal (-os), bientôt tombé (comme le prouve le glossaire de Vienne en gaulois tardif et latin évolué), reste un étymon qui, comme dans le cas du toponyme Cambo (Cambo-lesbains), passé tel quel sous sa forme neutre en basque par emprunt très ancien, est exactement leur terme emblématique argi (argi eta garbi), au sens de 'clair' ('clair et net'), avec une riche dérivation. Ce que

4. Xavier DelamarRe, Dictionnaire de la langue gauloise. Une approche linguistique du vieux celtique continental, Paris, Errance, 2001. 
n'a pas contredit un excellentissime spécialiste de lexicographie basque (l'ami Xarles Videgain) lorsque je lui ai posé la question! Et ce n'est là qu'un exemple de plusieurs dizaines d'emprunts qu'a pu faire l'euskera au celtique ancien à l'époque où ces langues étaient en contact, comme il le fit ensuite abondamment au latin, sans que cela en fasse une langue romane.

\section{Le pourquoi de cette étude}

Première réponse, il s'est agi comme ce fut également le cas dans le passé pour mes autres ouvrages (notamment mon dictionnaire bleu de 1992), d'une demande ou tout du moins d'une suggestion avec pression amicale du responsable des éditions associatives Skol Vreizh, Jean-René Le Quéau.

Suite au succès il y a quelques années des Bretonnismes d'Hervé Lossec, qui mettait en lumière - après d'autres, dont certains ici présents - le contact entre le breton jadis hégémonique et le français parlé en Basse-Bretagne, l'idée était de voir quelle avait été plus avant et plus largement l'influence du celtique sur le français et les autres langues qui l'entourent, romanes ou germaniques, etc.

Parallèlement je persévérais et continuais depuis une dizaine d'années, à mon rythme de retraité, de travailler sur mon Dictionnaire du breton contemporain, que j'ai mis en ligne récemment sur Internet (cf. ci-après), car à force d'ajouts divers il a atteint une telle taille qu'une publication papier me semble parfois problématique - même en plusieurs volumes, puisqu'il compte désormais quelque 4000 pages - d'autant que le fait de l'avoir sur la " toile" permet de continuer à l'améliorer.

Outre les corrections et les ajouts, à partir des travaux que j'ai eus à diriger à l'Université jusqu'à récemment (plusieurs thèses sur une douzaine), mais aussi grâce à l'apport de nombreux chercheurs et auteurs qui m'ont transmis spontanément tout ou partie de leur collecte lexicale (par exemple Daniel Giraudon pour le Trégor ou Yann Riou en Léon, mais aussi des Vannetais comme Raymond Le Bacon, sans compter des doctorants comme, entre autres, Loig Cheveau, Pierre-Yves Kersulec ou François Louis), je me suis intéressé à l'étymologie, celle du breton comme du français d'ailleurs.

Ceci répond également à une interrogation récurrente sur les origines celtiques de la petite Bretagne car le renouvellement des recherches préhistoriques ont amené plusieurs auteurs, faute de répondant linguistique, à douter haut et fort que la Bretagne ait jamais eu un peuplement qui fût " celtique ", ce qui, il faut bien le reconnaître, quelles que soient les théories ambiantes, laisse entière à leur débit la question des langues qui auraient préexisté en Ar- 
morique, à moins qu'elles fussent toutes coupées!

\section{Le comment de la démarche}

Cela m'amène à aborder le comment, après le pourquoi. Tout d'abord, je dois souligner qu'il s'agit là d'une méthode - si méthodologie il y a - ou, à dire tout net, d'une simple démarche empirique, partie du terre à terre, dans le cas présent d'une réalité toute prosaïque, celle des mots de tous les jours.

Ayant eu l'occasion de suivre jadis les cours de Léon Fleuriot durant mes études, j'avais une certaine connaissance de l'étymologie celtique de base, nourrie de moyen-gallois et d'un peu de vieil irlandais, telle que l'ont développée les philologues tout au long du XX ${ }^{\mathrm{e}}$ siècle et j'avais bien sûr pris connaissance des travaux de l'abbé Falc'hun, dont j'ai suivi quelques conférences plus tard, notamment sur la toponymie, ainsi que les travaux menés depuis lors par Pierre-Yves Lambert.

De même, je l'ai dit, j'ai pu utiliser les synthèses récentes, y compris en ligne, notamment à partir du remarquable dictionnaire de la langue gauloise de Xavier Delamarre, préface par notre collègue Lambert, qui a d'ailleurs été réédité avec quelques ajouts significatifs récemment mis en lumière.

Ces publications, qui sont à la fois un reflet de recherches pointues à partir des découvertes diverses qu'a permises l'archéolo- gie préventive notamment, mais aussi une vulgarisation auprès de publics lettrés très variés, ont un fort retentissement et un large écho sur Internet, dans la mesure où ils ont apporté des éléments solides pour la recherche des origines de nombreux toponymes, par exemple.

C'est donc cet ensemble de connaissances accumulées, des apports plus anciens de remarquables celtisants comme Dot$\operatorname{tin}^{5}$ aux nouvelles découvertes de termes épars, voire de phrases entières, parfois bilingues, amenant diverses réinterprétations d'éléments plus anciens... qui m'ont permis de réunir un matériau relativement étoffé.

Si l'on admet que le gaulois ou, disons plutôt désormais, le celtique continental n'est guère différent du celtique insulaire, surtout en premier lieu du britonnique ou, dit-on, du British (comme l'affirmait César en son temps), à côté du celtibère, dont le déchiffrement est loin d'être terminé, ou encore du galate tout récemment revisité par Philip Freeman, issu d'Harvard, auteur d'un précieux ouvrage, The Galatian language ${ }^{6}$

5. Georges Dоттіл, Langue gauloise, Paris, C. Klincksieck, 1918, accessible en ligne : http://gallica.bnf.fr/ark:/12148/ bpt6k2100481 (consulté le 18 juin 2018).

6. Philip Freeman, The Galatian language. A comprehensive survey of the language of the ancient Celts in Greco-Roman Asia Minor, Lewiston (N.Y.), Edwin Mellen press, 2001. 
avec comme sous-titre A Comprehensive Survey of the Language of the Ancient Celts in Greco-Roman Asia Minor (Ancient Near Eastern Texts and Studies). Il contient quelque cent vingt termes (beaucoup de noms propres antiques) écrits en alphabet grec, tous attestés en gaulois et généralement identiques (galate n'étant de fait que le vocable grec signifiant gaulois), pratiquement tous restés en breton comme en gallois (ou britonnique), avec des évolutions diverses bien sûr... alors nous, Bretons - bretonnants et un peu celtisants donc - ne sommes assurément pas les plus mal placés pour en parler !

En effet, la plupart de ceux qui aujourd'hui travaillent sur la matière la plus antique ont souvent une connaissance approfondie de ces langues celtiques anciennes, telles qu'elles ont été mises par écrit, vieil irlandais comme moyen-gallois, mais cela date quand même d'un bon millénaire plus tard, même si les gloses remontent avant l'an mil. Le continuum breton est dès lors des plus pertinents.

Pour reprendre un seul exemple, le glossaire de Vienne (dit d'Endlicher), qui contient une douzaine de termes en gaulois tardif glosé en latin, peut-être de la région de Bordeaux autour du $\mathrm{V}^{\mathrm{e}}$ siècle $^{7}$, nous fournit la forme

7. Voir Francis FaVereau, Babel et baragouin. Le breton dans la mondialisation, Morlaix, Skol Vreizh, 2006. treide (glosé en latin tardif " pede», soit cas final de " pied - issu de l'accusatif «pedem » / "pes»).

D'évidence, cette forme est extrêmement proche du pluriel breton actuel treid, pluriel interne de troad (troed gallois a pour pluriel la forme correspondante traed), alors que la forme en gaulois plus ancien était traget(qui a de même laissé des traces dans des mots dialectaux comme le "vautre").

D'un autre point de vue, toujours considéré côté breton, une des traces les plus anciennes qui soit en vieux-breton est une inscription sur une stèle du cimetière de Lanrivoaré en Léon (connu également pour sa légende des 7777 saints - un sept en moins serait peut-être plus conforme !) : Gallmau.

Les deux termes sont toujours très présents et bien attestés en breton de toute époque, davantage peut-être qu'en gallois d'ailleurs, mais aussi en irlandais, avec une palette de sens très large pour l'un comme pour l'autre, mais in fine sous forme de radicaux insécables comme noms propres (noms de famille, parfois aussi de lieu) - à savoir (Le) Gall \& (Le) Mao, ne serait-ce que plein les cimetières - alors qu'ils bénéficient d'une dérivation conséquente (pour ce dernier, outre l'adjectif mao, le patronyme Mao, le féminin maouez, le dérivé mewel / mevel, autant de patronymes très courants, de même qu'en composition Mau- 
bian, Maudu, Mauglas, Mavic, Mocaer \& Cosmao, Cosmao, \& Lemau etc., jusqu'à Kervodu ou Trévodu, lieu-dit à Poullaouen).

Mais on prétend que le terme, qui est passé en français populaire jadis pour désigner un marin (un "mao" ou même "sale mao", injure de “moko"), tant à Toulon qu'à Bizerte jadis, paraît-il, également usité en gallo avec le même sens (selon divers témoignages, écrits comme oraux très récents), aurait pu donner par l'intermédiaire d'une forme dialectale du grand Ouest le mot " ma(h)ous ", dont le sens - via celui de " gaillard »n'est pas si éloigné qu'il n'y paraît de celui de notre originel mao...

Où l'on réalise que, compte tenu de la perte des cas à cette haute époque - tant en latin qu'en celtique après la chute de l'Empire, dit-on - la frontière est ténue, voire quasi inexistante, entre gaulois tardif, tel que nous pouvons le deviner et le vieux-breton le plus ancien, tel qu'on ne le connaît que très peu également, puisque la plupart des gloses publiées datent davantage des $\mathrm{IX}^{\mathrm{e}}$ et $\mathrm{X}^{\mathrm{e}}$ siècles...

En filigrane, se confirme donc cette notion de continuité ou de continuum, sans reprendre à la lettre nécessairement, pour autant, celle développée ici même par Alieni, par exemple, cette continuità à l'italienne qui fait du celtique la langue des premiers habitants sédentarisés sur la façade atlantique.
Un autre élément essentiel dans cette recherche de filiation, par-delà les formes écrites parfois incertaines ou tout simplement absentes, reste ce qu'on appellera le "son ", c'est-à-dire une oralité qui a dû présider à ces emprunts et autres transmissions de boucheà-oreille, davantage que par écrit, même si le latin du clergé, naguère langue internationale et de culture, a pu jouer un certain rôle.

Prenons de nouveau un exemple particulièrement parlant.

Les lexicographes - auteurs des principaux dictionnaires et sites divers en ligne pour le français considèrent que le verbe français " craindre " a une origine celtique, quelque chose comme *crenare, peut-être influencé - selon Lambert - par la morphologie du latin «tremere" (d'où vient «trembler $»)$.

Mais plus prosaïquement, si on compare le français dans sa conjugaison (ça " craint " / " crainte " etc. - j'ignore là " craignos "!) on s'aperçoit qu'à l'oreille cela ressemble bigrement à la prononciation bretonne orientale / $\mathrm{kr} \tilde{\varepsilon}: \mathrm{n} /$ qui est, au moins, celle de l'est du Poher, à côté de l'allomorphe plus courante avec un E fermé (soit / krẽ:n/), apparenté au gallois qui l'emploie même pour les Quakers!

Un autre aspect est un certain va-et-vient ou, à tout le moins, un échange répété entre langues, celtique ancien, latin puis roman 
et breton, ici donc parangon des langues néo-celtiques.

Le même glossaire de Vienne contient un autre verbe - alors que ceux-ci sont réputés être nettement moins connus en celtique antique que les noms et adjectifs : cambiare, en latin rem pro re dare (glose : donner une chose pour une autre avec le sens d'échange autant que change donc).

Si cambio est passé tel quel dans les nombreuses langues romanes, il a bien évolué en français, jusqu'à la forme " change ", laquelle est repassée en moyenbreton sous des formes assez diverses (d'abord "cench", cheñch \& chañch, chañj - encore en vannetais), alors qu'en ligne plus directe elle s'est transmise en breton sous le vocable plus rare $\mathrm{kemm}$, également attesté ailleurs en néo-celtique.

Et c'est, comme je l'ai mentionné plus haut, à partir d'une forme apparentée (plurielle), «cambion " (dans "reguccambion», analysé par Lambert) - dont Cambo garde trace, par ailleurs, ainsi que moult Cambords ou Chambord, etc. que j'ai induit, par pure intuition je dois l'avouer, que l'on pourrait peut-être tenter d'expliquer «camion " (d'origine inconnue) par ces dites " courbes " que sont les bras de l'ancêtre de ce véhicule de transport, à l'instar des bordés en breton maritime, koad-kamm!

\section{Les limites du genre}

Quelle est, d'un point de vue purement quantitatif, la proportion d'items concernés?

Le nombre de mots faciles à rapprocher entre breton et gallois, sans parler du cornique qui se situe un peu dans un espace géolinguistique intermédiaire $\left(5^{\mathrm{e}}\right.$ dialecte), est de l'ordre de cinq mille.

C'est ce que j'avais déjà illustré dans mon dictionnaire bleu. Pour l'irlandais-gaélique, Eamón Ó Ciosáin en avait repéré environ trois mille, qui ont été incorporés au dictionnaire bleu également (certains précisés depuis par Loig Cheveau pour la version plus récente mise en ligne).

Ce chiffre - 5000 mots - est, par ailleurs, celui du registre normal des locuteurs dans la plupart des langues, si l'on excepte l'usage érudit, professionnel ou savant, qui se rapporte davantage à l'écrit ou parfois (en médecine, par exemple) à une nouvelle lingua franca (faite de latin et surtout de grec). Les jeunes dits en difficulté (scolaire ou sociale) ont d'ailleurs un corpus linguistique très inférieur, même si les argots ou parlers divers complètent souvent un usage de la langue de base assez réduit. Autant dire que ce nombre correspond à un usage moyen, y compris du breton contemporain, puisque c'était là celui du lexique de ma thèse sur le breton dit « central» (du Poher). 
On remarquera que le Catholicon, premier dictionnaire bien connu, contient également environ 5000 entrées. Mais même si le breton de Lagadeuc ne diffère pas considérablement dans ses termes basiques du breton tel qu'il subsiste sur les mêmes terres (au sud de Morlaix, tout près du Poher en l'espèce, sorte de continuum Morlaix-Plougonven-ScrignacPoullaouen-Carhaix, voire SaintHernin pour "Quoaet-queveran», de l'Arrée à l'Aulne), il ne s'agit pas exactement - mutatis mutandis - de la même nomenclature, comme on peut s'en douter, le monde ayant légèrement bougé depuis 1499 !

Pour ce qui est de la période précédente, à titre de comparaison, on peut considérer que les gloses du vieux-breton (au sens que lui donnait Loth, comprenant également vieux cornique et gallois), étudiées surtout par Fleuriot, contiennent quelque deux mille termes, mais glanés à partir des seuls manuscrits du haut Moyen Âge, datant des alentours de l'an mil ou après, faut-il le rappeler?

Enfin, si l'on prend le récent dictionnaire du gaulois de Delamarre, il contient près de mille entrées. Mais si on se réfère à ses annexes et à d'autres publications, basées sur l'anthroponymie et la toponymie où abondent les composés et parfois les dérivés, on atteint des chiffres comparables.

À titre de comparaison, le seul galate - d'Asie centrale ou, pour simplifier de Galatasaray (sérail galate, soit palais gaulois) - connu essentiellement par des noms propres, compte selon Freeman cent vingt termes, dont tous se retrouvent logiquement en gaulois, qui était la même langue.

Nous avons donc là, au moins sur le continent - on n'ose dire l'Eurasie ! - un continuum d'un gros millier d'items que l'on peut qualifier de millénaires, formant l'ossature de ce qu'a pu être le celtique.

Il faut garder à l'esprit qu'à partir d'un étymon avéré (par exemple «barro- » pour «barre » et «bar »... passé jusqu'en arabe tunisien, ou " carros " pour " car " également etc.), nous avons, ne serait-ce qu'en français, des efflorescences lexicales qui rempliraient à l'envi des colonnes, voire des pages des dictionnaires, sans compter les composés et dérivés (ex. «braie»d'un « braccae » - dans les verbes " débrayer » ou " embrayer ", sans oublier la " braguette "... tout comme " combre "- d'un comberos - dans "décombres " et " encombre ", de même qu'en espagnol escombros, comme je le lisais suite au terremoto de Mexico...)

Ceci étant dit, le différentiel avec l'ensemble du breton des dictionnaires - quelque 50000 mots - s'explique soit par la dérivation devenue exponentielle désormais (dont la suffixation abondante qui explique aussi la divergence croissante avec le gallois, par exemple), 
soit par les emprunts, parfois intempestifs d'ailleurs, faits aux langues en contact, alors que les variantes anciennes comme le vieil irlandais contiennent une masse de mots qui n'ont généralement laissé aucune trace ailleurs.

Beaucoup d'inscriptions restent à expliciter en celtique antique, notamment en celtibère (Bottorrita). Car, de fait, seules les langues dites néo-celtiques permettent généralement d'interpréter des termes et parfois des phrases dont le sens sinon peut nous échapper totalement et peut-être définitivement.

Comme quoi c'est encore « nous »- Bretons et Britons, etc. - qui restons témoins du fonds gaulois! 